\title{
Cáncer y la pobreza están fuertemente correlacionados en el Perú
}

\section{Cancer and poverty are strongly correlated in Peru}

\author{
Milward Ubillús $s^{1,2, a}$
}

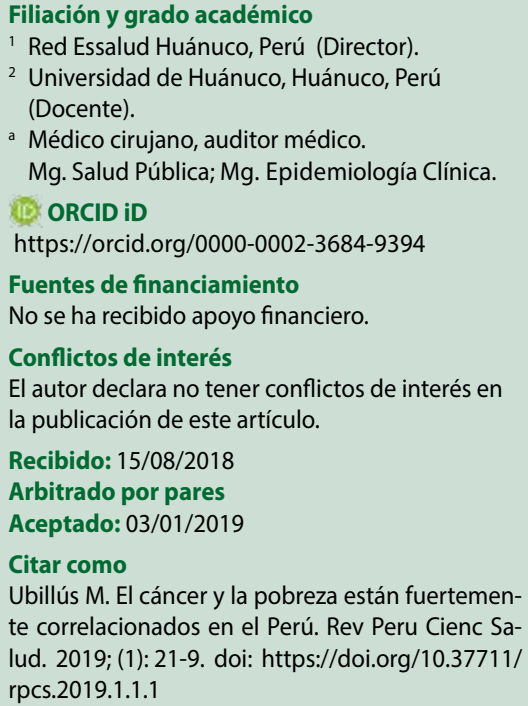

\section{RESUMEN}

Esta es una investigación ecológica retrospectiva de múltiples grupos, cuyo objetivo es encontrar la correlación entre la vulnerabilidad al cáncer y la pobreza en el Perú durante el 2013. En la metodología, se utilizó el muestreo de conveniencia no aleatoria, incluidos todos los departamentos del Perú. Las principales fuentes utilizadas para el análisis fueron: la situación del Cáncer en Perú, 2013, del Ministerio de Salud, y los datos de pobreza del país, del Instituto Nacional de Estadística e Informática para el mismo año (2013); ya que estas son las últimas en publicaciones de datos nacionales sobre el cáncer y la situación de pobreza en Perú, respectivamente. Para la validación de los rangos para prueba de hipótesis estadística se utilizó la correlación de Spearman. Los resultados muestran una fuerte correlación estadística de 0,761 directa y significativa entre la vulnerabilidad al cáncer y la pobreza en Perú, durante 2013. Asimismo, la tasa y el alcance de una mayor vulnerabilidad al cáncer en las regiones del Perú, y jerárquica identificada como "Análisis del estado del cáncer en Perú - 2013", por Ministerio de Salud es: Huánuco, Ayacucho, Huancavelica, Cajamarca, Amazonas, Loreto y Pasco, todos muy vulnerables. Se concluye indicando que existe una alta correlación entre la vulnerabilidad al cáncer y la pobreza en Perú en 2013.

Palabras Clave: cáncer, Estudios retrospectivos, Pobreza Perú, Ambiente, Academias e Institutos, Publicaciones, Neoplasias (fuente: DeCS BIREME).

\section{ABSTRACT}

This is a retrospective ecological research of multiple groups, whose objective is to find the correlation between vulnerability to cancer and poverty in Peru during 2013. In the methodology, non-random convenience sampling was used, including all departments of Peru. The main sources used for the Analysis were: The Situation of Cancer in Peru, 2013, of the Ministry of Health, and poverty data of the country, of the National Institute of Statistics and Informatics for the same year (2013); since these are the latest in national data publications on cancer and the poverty situation in Peru, respectively. Spearman's correlation was used to validate the ranges for testing the statistical hypothesis. The results show a strong statistical correlation of 0.761 directly and significantly between cancer vulnerability and poverty in Peru, during 2013. Likewise, the rate and extent of greater vulnerability to cancer in the regions of Peru, and hierarchical identified as " Analysis of the state of cancer in Peru - 2013 ", by the Ministry of Health is: Huánuco, Ayacucho, Huancavelica, Cajamarca, Amazonas, Loreto and Pasco, all very vulnerable. The main conclusion is that there is a high correlation between vulnerability to cancer and poverty in Peru in 2013.

Keywords: retrospective Studies, Poverty Peru, environment Academies and Institutes, Publications, Neoplasms (source: MeSH-NLM). 


\section{INTRODUCCIÓN}

El cáncer es un problema de salud global, evidenciado por los altos indicadores epidemiológicos que presenta, los cuales se hallan en crecimiento. Este problema aparece en los países pobres y en crecimiento, de similar forma que en los del primer mundo ${ }^{(1,3)}$. Incluye un grupo de patologías que afecta a toda la población en el ámbito mundial y nacional, no discrimina edad, sexo, grupo racial, condición social, nivel económico ${ }^{(1,4)}$.

Se estiman 12,7 millones de casos nuevos de cáncer por año ${ }^{(1,2,3)}$ sin avizorar su disminución. Se pronostica para el 2030 un incremento a 21,3 millones de nuevos casos de cáncer ${ }^{(1,3)}$. A causa de ésta letal enfermedad, la Organización Mundial de la Salud (OMS) calculó 7,6 millones de muertes en 2005 y pronostica para los próximos 10 años, la nefasta suma de 84 millones de muertes, si no se interviene y aborda el problema ${ }^{(1,3,14)}$. Cifras mayores de los $2 / 3$ de dichos fallecimientos ocurrieron en países con ingresos medios y bajos, donde existen escasos o nulos presupuestos para poder trabajar en prevenición

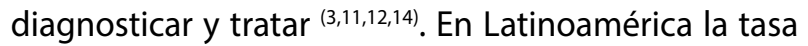
estandarizada por edad es de 163 casos por cada 100 000 habitantes, menor que en USA donde se tiene 300 casos por cada 100000 habitantes y menor que en Europa que presenta 264 casos por cada 100000 habitantes. Sin embargo la mortalidad es mayor que en USA y Europa; hecho atribuido al diagnóstico en estadios avanzados, dado la dificultad en el acceso a la salud en éstos países de menores recursos ${ }^{(3,13,14)}$.

GLOBOCAN $2008^{(3)}$, de la Agencia Internacional de Investigación en Cáncer (IARC), estima la incidencia acumulada para el Perú en 157,1 casos por 100 000 habitantes, menor en la población masculina que la femenina, 140,9 por 100000 vs. 174,9 por 100000 , entonces se diagnosticarían en nuestro país, aproximadamente 45000 casos nuevos de cáncer por año ${ }^{(1,3)}$. El Registro de Cáncer de Lima Metropolitana del Instituto Nacional de Enfermedades Neoplasicas (INEN), estimó para el período 2009-2011, 34000 casos ${ }^{(15)}$.

El Análisis de la Situación del Cáncer en el Perú 2013 (ASIS Ca Perú, 2013) (1), al analizar la topografía y el sexo de los pacientes, halló los siguientes tipos de cánceres según frecuencia: $14,9 \%$ para cuello uterino, $11,1 \%$ para estómago y 10,3\% para mama. Al hacer la discriminación según sexo, reporta para varones: $15,1 \%$ para prostata, $15,1 \%$ para estomago, y $7,8 \%$ para piel. Por otro lado, en mujeres aparecen:
$24,1 \%$ para cérvix, 16,6 \% para mama y 8,6 \% para estómago ${ }^{(5)}$. Concluyendo que el cáncer es un problema de principal prioridad, en la salud pública del país, representando la segunda causa de muerte ${ }^{(1,13)}$. El Registro de cáncer de Lima Metropolitana 2004$2005^{(15)}$, reporta el cáncer de mama como el de mayor tasa de incidencia, hallando una incidencia de $17,8 \%$ y una mortalidad de 5,2 \%, también halló para el cáncer de cérvix tasas altas y preocupantes con una incidencia $10 \%$ con una mortalidad de $4,1 \%$, de igual forma según el último reporte del Perfil Epidemiológico del INEN 2000-2012 ${ }^{(16)}$, los dos tipos más frecuentes de nuevos casos de cáncer en esos doce años, son el cáncer de Cuello uterino, con un $16,1 \%$ y el de mama con $12,07 \%{ }^{(3,16)}$.

A nivel global, los especialistas diagnostican alrededor de 12,7 millones de casos nuevos de neoplasias ${ }^{(1)}$. En el 2030, se pronostica que habrá un incremento a 21,3 millones, en Latinoamérica se habrán diagnosticado 1,7 millones de nuevos casos y habrán fallecido un millón de pacientes por esta enfermedad ${ }^{(12)}$. A nivel mundial, el ratio de mortalidad sobre la incidencia por las neoplasias es mayor en los países que cuentan con recursos bajos que en aquellos países con altos. En el 2002 los países de bajos ingresos presentaron un $74,5 \%$; es decir 1,6 veces más a la frecuencia de presentación de los países de economías sólidas y estables que tuvieron un $46,3 \%$.

En Latinoamérica y el Caribe es 0,59 en comparación con el 0,43 de Europea y 0,35 de USA ${ }^{(11,12)}$. En el Perú las neoplasias son la causa número dos de muertes, representando el $18 \%$ de la mortalidad $(1,4,13)$, siendo la incidencia anual de 150,7 casos por 100,000 habitantes, aproximadamente para el $2012{ }^{(15,22)}$. La tendencia global actual se centra en la protección del paciente y la cobertura financiera total asumida por el estado, toda vez que es una enfermedad que se encuentra en expansión, atacando a poblaciones vulnerables y pobres, a las cuales deja prácticamente en la miseria ${ }^{(14)}$.

Latinoamérica parece que va en ese sentido; Perú, Brasil, México, Cuba, Argentina, Chile y Colombia a pesar de tener diferentes modelos sanitarios, disimiles en estructura y organización, comparten la realidad. En otras latitudes, los países emergentes tienen el mismo problema, siendo el Africa Sud Sahariana la que sufre el mayor impacto ${ }^{(10)}$. El costo total que conllevó ésta enfermedad en todo el planeta, considerando todos los procesos e intervenciones que 
se realizan para afrontarlo, en el año 2009, ascendió a USD 286 mil millones aproximadamente ${ }^{(14)}$. El 84,7\% de la población mundial, reside en países pobres y de medianos ingresos. Estos países presentaron el 61,3\% de los casos nuevos de neoplasias a nivel global, sin embargo sólo representaron el 6,2 \% de dichos gastos ${ }^{(14,26)}$. Todo lo anterior refleja los elevados costos que se deben asumir si se tiene que enfrentar una enfermedad de alto costo como es el cáncer.

En nuestro país, antes del año 2011 se tenían tres centros a los cuales se les asignaba recursos públicos para el manejo de ésta epidemia; entre el año 2011 y 2012 se logró la participación de los veinticinco gobiernos regionales y es en el 2014, cuando se ha alcanzado el mayor número de Unidades ejecutoras que desarrollan actividades de prevención y control del cáncer afectando presupuesto público; siendo una expansión paulatina y total hasta llegar a los niveles de atención primaria, atendiéndose financieramente la totalidad de la estructura del sistema sanitario peruano ${ }^{(13,17)}$. La tendencia global actual se centra en la protección del paciente y la cobertura financiera total asumida por el estado. Latinoamérica parece que va en ese sentido; Perú, Brasil, México, Cuba, Argentina, Chile y Colombia, a pesar de tener diferentes modelos sanitarios, disimiles en estructura y organización, cuyo único patrón homogéneo es que son sistemas fragmentados, avanzan en ese sentido asumiendo los costos financieros con fondos separados $y$ diversos ${ }^{(14,21)}$.

Perú es un país que tiene 30 millones de habitantes, aproximadamente, con una estructura y organización del sistema de salud fragmentado y segmentado, con un nivel de gasto inferior en $2,5 \%$ del PBI al promedio latinoamericano ${ }^{(23)}$. Perú a pesar de tener el nivel de gasto inferior al promedio del PBI de los países latinoamericanos ha demostrado que podemos integrar el sistema desde el sector público y otorgar una cobertura integral del paquete de enfermedades oncológicas, de manera plural y equitativa (24). Se planteó como objetivo hallar la correlación entre la vulnerabilidad por cáncer y la pobreza en Perú, en el período 2013.

\section{MÉTODOS}

Se realizó un estudio descriptivo, ecológico, de grupos múltiples, retrospectivo, transversal. Se usaron fuentes de datos primarios ${ }^{(32,34)}$. La población considerada para el estudio fueron 26 regiones geopolíticas del Perú, que suman 26, seleccionados por un tipo de muestreo no aleatorio, por conveniencia.
Para recolección y organización de datos; inicialmente fueron registrados con el instrumento de recolección de datos, luego fueron codificados según su jerarquización y registrados en una base en formato Excel, posteriormente se evaluaron con el Software STATA 12,0. En el análisis e datos se usaron las medidas de tendencia central y frecuencia porcentual ${ }^{(31)}$, para la contrastación de las hipótesis se usaron los rangos (correlación) de Spearman ${ }^{(30)}$.

\section{VARIABLES}

\section{Cáncer}

Se define como un conjunto de enfermedades relacionadas, las cuales en todos sus tipos, se dividen descontroladamente y sin detenerse, diseminándose a los tejidos de alrededor y los órganos distantes. Puede iniciarse en cualquier célula del organismo ${ }^{(18,21)}$. Conforme las células se van malignizando, aquellas dañadas a nivel genético, permanecen vivas y reproducen células hijas cuando no son necesarias, de manera indiscriminada. Estas células hijas adicionales se dividen y formar masas que se denominan tumores ${ }^{(18)}$.

\section{Pobreza}

Se entiende así a aquella condición de insatisfacción de las necesidades principales en comparación al nivel medio de satisfacción de la sociedad ${ }^{(2,29)}$. El bienestar individual o familiar depende del nivel de consumo o del nivel de los ingresos económicos, medidos en comparación a los estándares mínimos compatibles con la dignidad humana; lo que implica la satisfacción suficiente de las necesidades esenciales ${ }^{(2,29)}$.

\section{Aspectos éticos}

No hubo problemas éticos ni morales en el desarrollo de la presente investigación, ya que es un estudio retrospectivo, donde las unidades de análisis son las regiones y sus registros e indicadores. No existieron conflicto de intereses entre el autor y las instituciones de donde se registran los datos de la investigación. La investigación cumple las recomendaciones del Tratado de Helsinsky.

\section{RESULTADOS}

Respecto a la vulnerabilidad por cáncer y la pobreza, se registró un coeficiente de correlación de 0,761 y una $p \leq 0,001$, mostrando significancia estadística en la relación entre la vulnerabilidad por cáncer y la pobreza (ver tabla 1).

En relación a la dispersión de los puntos, se observa una relación positiva ascendente afirmando 
Tabla 1. Correlación entre la vulnerabilidad por cáncer y la pobreza en el Perú durante el año 2013

\begin{tabular}{lcc}
\hline Correlación & Valor & $\mathbf{p}$ \\
\hline Estadístico Rho de Spearman & 0,761 & $\leq 0,001$ \\
\hline
\end{tabular}

que mientras el índice de pobreza monetaria asciende también lo hace el índice de vulnerabilidad por cáncer (ver figura 1).

En cuanto al índice de vulnerabilidad por cáncer en el Perú, los departamentos con mayor índice fueron Huánuco y Ayacucho y los de menor índice fueron Arequipa y La libertad (ver figura 2).

Según grado de vulnerabilidad por cáncer en el Perú, los departamentos con mayor vulnerabilidad para cáncer fueron Huánuco, Ayacucho, Huancavelica, Amazonas, Loreto y Pasco lo que implica que requieren intervención inmediata; mientras que, y en condición de vulnerabilidad se encontraban los departamentos de Piura, Cusco, Tumbes, Apurímac, Puno y San Martín, lo que implica que requieren fortalecerse en el cortomediano plazo (ver figura 3).

Concerniente al índice de pobreza monetaria en el Perú, los departamentos de Cajamarca, Ayacucho, Amazonas, Huancavelica, Pasco, Apurímac y Huánuco superaron la tasa del $40 \%$. En cambio, los departamentos de Arequipa, Moquegua, Ica y Madre de Dios tuvieron tasa de pobreza más baja, menos del $10,0 \%$ (ver figura 4 ).

En relación al mapa de pobreza monetaria, en el primer grupo con incidencia de pobreza más alta se ubicaron cinco departamentos: Cajamarca, Ayacucho, Amazonas, Pasco y Huancavelica. En el segundo

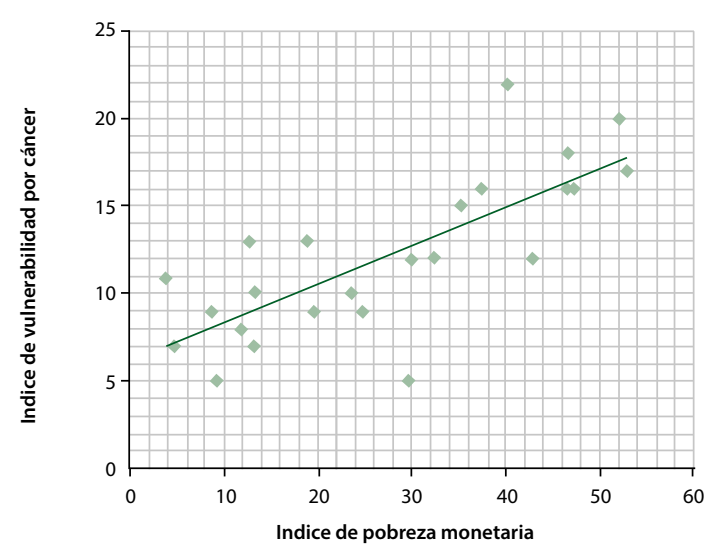

Figura 1. Correlación entre la vulnerabilidad por cáncer y la pobreza en el Perú durante el año 2013

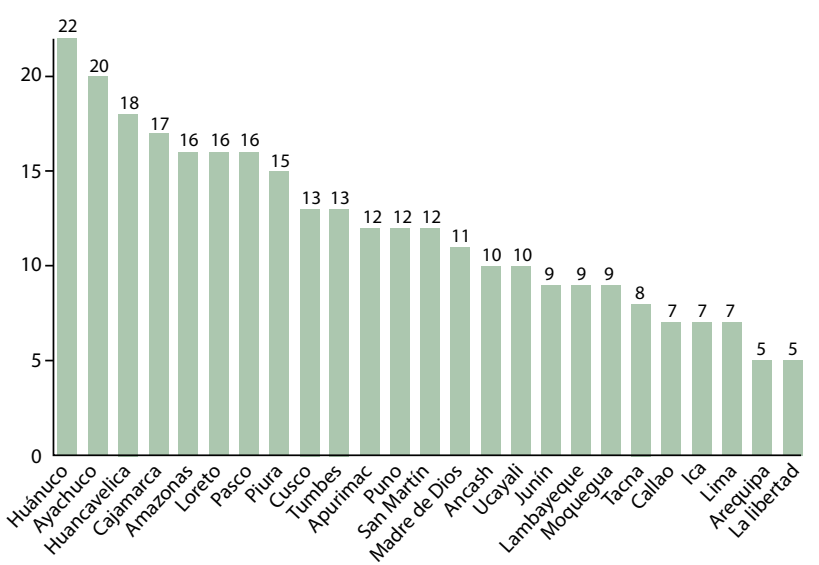

Figura 2. Índice de vulnerabilidad por cáncer en el Perú, 2013

grupo estuvieron: Apurímac, Huánuco, Loreto y Piura. El tercer grupo de departamentos lo integraron: La Libertad, Puno y San Martín. En el cuarto grupo se encontraron los departamentos de, Áncash, Cusco, Junín y Lambayeque. El quinto grupo lo conformaron: Provincia Callao, Provincia Lima, Región Lima, Tacna, Tumbes y Ucayali. En el grupo sexto estuvieron: Arequipa, Moquegua. Y, en el último grupo con la tasa de pobreza más baja, se encontraron Ica y Madre de Dios (ver figura 5).

\section{DISCUSIÓN}

La presente es una investigación ecológica de grupos múltiples y retrospectiva ${ }^{(35)}$, donde los grupos estuvieron conformados por las regiones del Perú. Nuestros resultados demuestran una alta correlación estadística directa entre la vulnerabilidad por cáncer y la pobreza en el Perú; con lo que se reforzaría la teoría de la relación entre el cáncer y pobreza. Se tomaron datos de fuentes primarias como el documento Análisis de la Situación del Cáncer en el Perú, 2013,

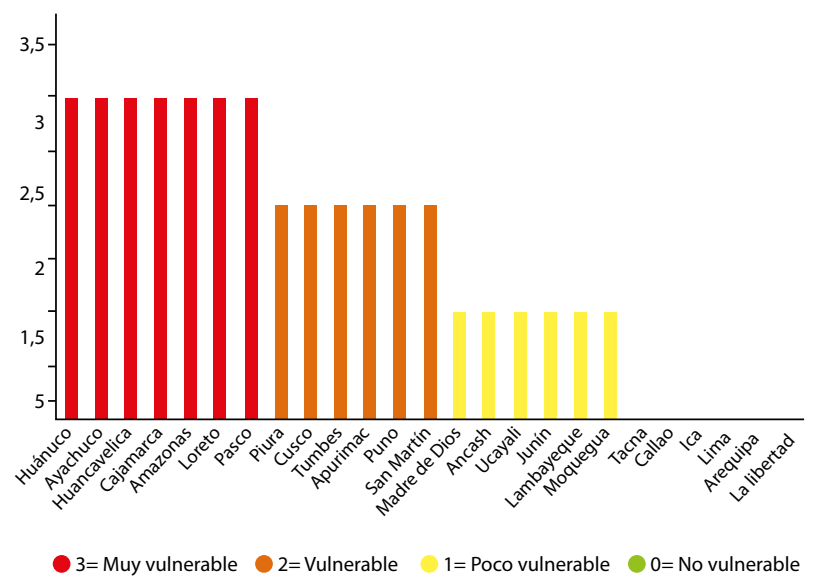

Figura 3. Grado de vulnerabilidad por cáncer en el Perú, 2013 


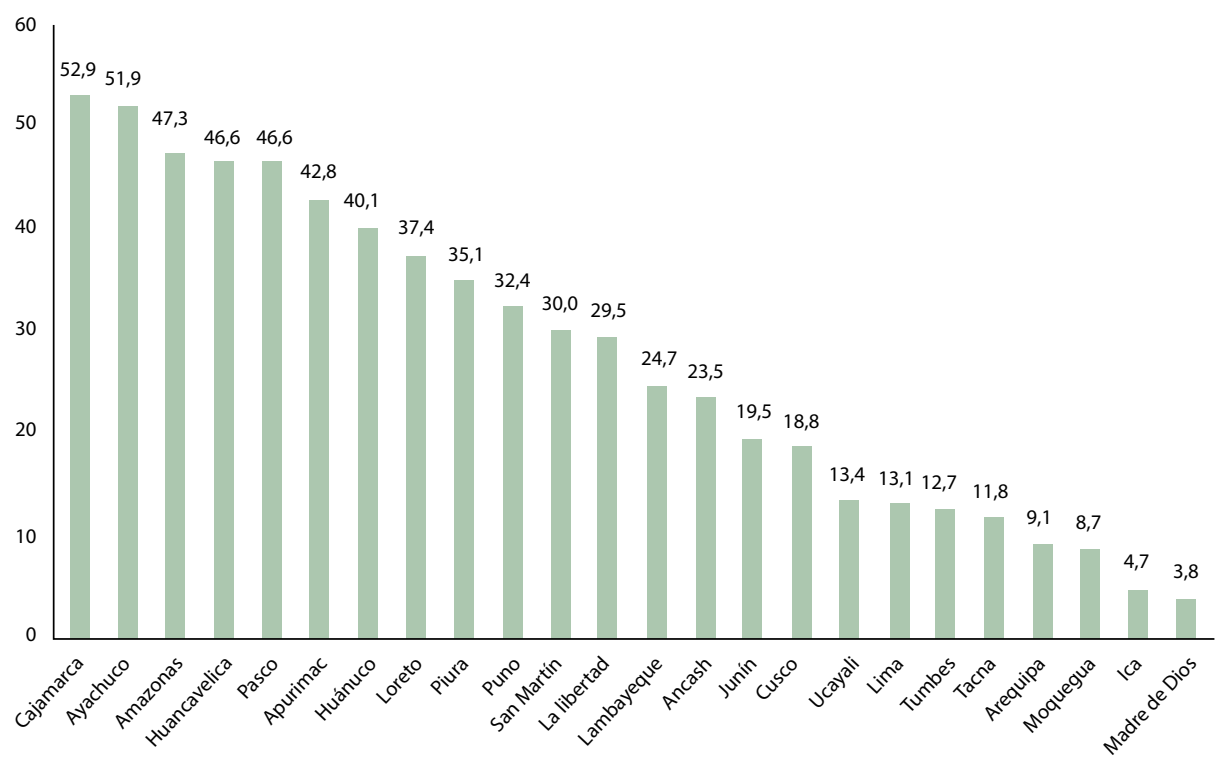

Figura 4. Índice de Pobreza Monetaria en el Perú, 2013

del Ministerio de Salud ${ }^{(1)}$; toda vez que es el último que publica datos nacionales sobre la situación del cáncer en el Perú por otra parte los datos de pobreza del país fueron tomados del Instituto Nacional de Estadística e Informática para el mismo año (2013). El Globocan - IARC, con las cifras que publicaron en la última década, ha desmitificado el hecho de que las neoplasias son un problema de los países del primer mundo y las enfermedades infecciosas de los países del otro extremo ${ }^{(3,12)}$. Según las instituciones referidas, de los 11 millones de neoplasias que se produjeron el 2008 en el planeta, más del $50 \%$ se reportaron en pacientes del tercer mundo, y más del $66 \%$ de los 7,6 millones de fallecimientos se dieron en los países en fase de desarrollo y pobres ${ }^{(4,21)}$. Un número importante de factores están implicados en las neoplasias en los estratos más bajos desdela visión socioeconómica ${ }^{(11,21)}$; lo que nos demostraría la importancia de entender el problema, con una mirada de los determinantes sociales, donde los pobres son los más afectados. El
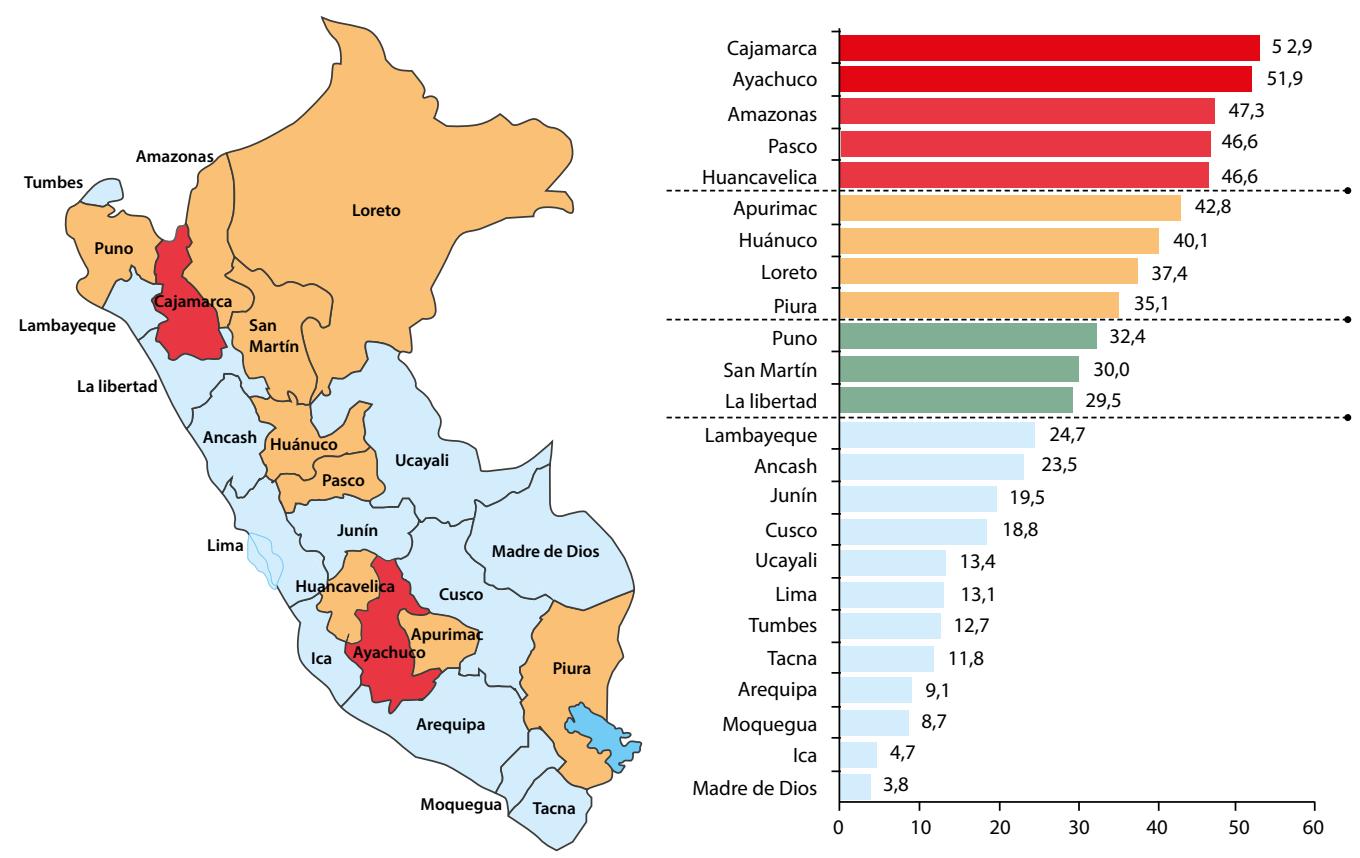

Figura 5. Mapa del Índice de Pobreza Monetaria en el Perú, 2013 
resultado hallado confirma lo que Solidoro dice en su artículo sobre la pobreza, Inequidad y Cáncer ${ }^{(4)}$, que el gran desafío del control del cáncer en el siglo XXI, es reducir la morbimortalidad por este mal en países en desarrollo y nuestro estudio relaciona la pobreza y el cáncer.

En el mundo se diagnostican aproximadamente 12,7 millones de casos nuevos de neoplasias y se calcula que para el año 2030, se incrementará a 21,3 millones de casos nuevos. En América Latina se habrán diagnosticado 1,7 millones de casos nuevos y habrán fallecido un millón de sujetos por esta enfermedad. ${ }^{(1,3)}$. Al comparar lo hallado en la presente investigación con el estudio de Gutiérrez C. y Alarcón E. ${ }^{(5)}$ en su trabajo "Nivel de pobreza asociado al estadio de gravedad del cáncer ginecológico, INEN 2004 -2005", pudimos demostrar la misma hipótesis, solo que en ésta investigación, la relación neoplasias - pobreza, se realizó a nivel global de todos los tipos de cánceres, determinando también una correlación directa fuerte entre ambas variables.

Al igual que Benites, Rodríguez, Mezones ${ }^{(6)}$; en su estudio "Determinantes sociales del cáncer de cuello uterino", publicado por el Instituto Nacional de Salud en Diciembre del 2013, reafirmamos el postulado de que existe relación entre los determinantes sociales y el cáncer de cérvix, ampliando el espectro estudiado a todas las neoplasias; dado que la mortalidad por esta causa se ha incrementado en nuestro país de forma preocupante grave en las últimas décadas. Cuando contrastamos las conclusiones de Andrés Solidoro de su investigación "El Cáncer Gástrico y la pobreza" desarrollado en el Instituto Oncológico de Lima (7) el perfil epidemiológico de cáncer de las regiones más pobres, muchas de las cuales están ubicadas geográficamente en la sierra de nuestro país, son similares al presentar entre los tipos de neoplasias, el cáncer gástrico como la primera causa de muerte por ésta enfermedad, siendo muy preocupante su afirmación de que "tres mil de las nueve mil muertes anuales por cáncer de nuestro país se deben a éste tipo, desproporción elevada con el 8,9\% del total de fallecimientos por cáncer gástrico en el mundo" (7).

La presentación de la enfermedad en su forma de cáncer gástrico, como la más prevalente en algunas regiones del país, tales como Huánuco (29\%), Huancavelica (19 \%), Ayacucho (16\%) y Junín (14\%) sugiere que son los ciudadanos más pobres las víctimas más frecuentes de éste tipo de enfermedad.
Samuel Arias en su publicación, Inequidad y Cáncer - una revisión conceptual ${ }^{(8)}$ dice: "en general, las desigualdades en salud entre los diversos grupos sociales, son injustas ya que reflejan una inequitativa distribución, de los determinantes sociales, tales como la educación, el trabajo, los servicios de salud, discriminación por raza, género y nivel socioeconómico, entre otros lo cual se puede evidenciar también en nuestra realidad" (8). La realidad que expresa Chan Margaret, en otro artículo, titulado "En África, el cáncer cobra más vidas por la pobreza" (10), al decir que menos del $15 \%$ de pacientes con cáncer de cérvix y mama, altamente curables en otros países, tienen una tasa de sobrevida de cinco años, nos recuerda nuestros estratos marginales y de extrema pobreza del Perú ${ }^{(10)}$.

Lo mismo se evidencia en estos grupo poblacionales y regiones del Perú, cuando describe que "la mitad de países del continente africano, carecen de máquinas de radioterapia", toda vez que en todo el país solamente contamos con tres máquinas de radioterapia operativas en la fecha de estudio; así como que tampoco tienen equipamiento, infraestructura, ni personal capacitado para afrontar las demandas a largo plazo de las atenciones de las neoplasias. Como se puede ver el continente más pobre del mundo presenta las tasas epidemiológicas más graves en cáncer, con lo cual se puede inducir la relación entre el cáncer y la pobreza, demostrada en la presente investigación.

La nota de la OMS titulada, "La batalla mundial contra el cáncer no se ganará únicamente con tratamiento"(11), cobra total validezen nuestro contexto, toda vez que los costos asumidos son altos por la carga de cáncer, estando lejos de la capacidades de poder asumirlos por parte de los países en desarrollo, como el nuestro y ejerciendo una presión grande sobre nuestro sistemas sanitarios ${ }^{(11)}$. Purilla Stephanie, asesorada por Ubillús Milward, en su tesis titulada "Correlación entre el Cáncer Ginecológico y el Nivel socioeconómico. INEN 2010-2012" (12), que presenta resultados hallados que indican una correlación positiva, de 0,813, por el estadígrafo de Spearman, para una $p<0,001$ lo que nos demuestran que existe una fuerte correlación directa, estadísticamente significativa entre las variables investigadas, siendo los resultados muy similares a los nuestros y reforzando, por tanto nuestra teoría de asociación entre el cáncer y la pobreza en nuestro país. 
Nuestros resultados nacionales, se refuerzan con las estadísticas del Registro de Cáncer - Lima Metrópoli 2004 - $2005^{(15)}$, calculado, por Payet Eduardo, Pérez Patricia, Poquioma Ebert y Ubillús Milward, quienes presentan evidencia de la relación entre los grados de pobreza y las neoplasias según estratos en la capital peruana. Ubillús Milward y colaboradores en el resumen titulado “Perfil Epidemiológico del Cáncer en el Instituto de Enfermedades Neoplásicas, Perú. 20002011"(16), presentado en el 5. ${ }^{\circ}$ Congreso Internacional del Control de Cáncer, desarrollado en Lima en noviembre del 2013, nos presenta su investigación epidemiológica, descriptiva y retrospectiva, que se reportan 109251 casos nuevos diagnosticados de cáncer, para ese período de tiempo, discriminando como los diez tipos más frecuentes, a los cáncer de cérvix $(16 \%)$, mama $(12,1 \%)$, estómago $(8,1 \%)$, leucemias $(5,6 \%)$, próstata $(4,6 \%)$, piel no melanoma $(4,4 \%)$, pulmón $(4,2 \%)$, colorrectal $(4,1 \%)$, linfomas $(3,6 \%)$ y tiroides $(3,1 \%)$ Según su género, el femenino presentó el $63 \%$ de los casos, mientras que el masculino el $37 \%{ }^{(16)}$.

En cuanto a grupos de edades, se evidencia que el comprendido entre los 41 y 60 años presenta la mayor proporción de casos con 37,5 \% del total. La condición social predominante fue la Hospitalaria (55,2\%) seguida de la condición SIS (Seguro Integral de Salud) $(14,3 \%)$, que se incrementa más en los últimos años y Essalud (Seguridad Social) $(12,8 \%)^{(16)}$; donde se puedo evidenciar que las condiciones sociales más pobres son las que tienen las mayores frecuencias de cáncer. Estos resultados reflejarían un perfil importante que se asocia con la pobreza de la población atendida en el INEN; un grupo significativo de la realidad peruana.

Ubillús, et al en el resumen de su investigación sobre"El Modelo lógico-causal y la Medicina Basada en Evidencia para el cáncer e Intervenciones Sanitarias del $\mathrm{PPR}^{\prime(19)}$, presentan un modelo de diseño que establece relaciones de los productos según plazos (de corto, medio y largo) con las actividades, que se a su vez se relacionan con los procesos y los aspectos teóricos, los cuales se apoyan en evidencia científica. Este diseño realizado por el autor de la presente investigación busca hacer un uso eficiente de los recursos en el "Programa presupuestal de cáncer", el cual es medido por sus resultados, buscando principalmente llegar a los más pobres, como se ha demostrado en los últimos años. En Perú las neoplasias ocupan el segundo lugar, como causa de fallecimiento, representando el $18 \%$ de la mortalidad ${ }^{(1,13)}$.
Según el INEN, institución responsable del control y manejo del cáncer a nivel nacional, la tasa de incidencia anual (2012) se estima en 150,7 casos x 100000 habitantes. 45000 casos incidentes y 80 000 casos prevalentes. ${ }^{(1,13)}$. La pobreza o incluso un grado socioeconómico inferior estarían vinculados causal y directamente con una elevada tasa de incidencia de las neoplasias, por lo que se convierte en el determinante social más importante de ser abordado y atendido. ${ }^{(11,20)}$. Las mujeres pobres tienen pocas opciones de hacerse un examen clínico de descarte y complicaciones en el acceso al tamizaje. Así, en el caso del cáncer de cérvix, tienen dificultad para acceder a un Papanicolaou o a poder realizarse una mamografía ${ }^{(11,20)}$, mientras que las mujeres no pobres, tenían el doble de posibilidades de someterse a un Papanicolaou y casi el triple de hacerse una mamografía ${ }^{(14,26)}$. Por otro lado es importante destacar que existen barreras y dificultades para aumentar el volumen poblacional para el control del cáncer en prevención primaria y secundaria en todos los niveles socioeconómicos en los países pobres ${ }^{(6,7)}$.

Las neoplasias, particularmente algunas de sus tipos, están fuertemente relacionados a la pobreza; entre ellas la mortalidad por cáncer gástrico, de cérvix y de pulmón, siendo mayor en los estratos sociales bajos que en los de la clase media ${ }^{(4,8,27)}$. El caso del cáncer cérvix constituye ejemplo de éste duro drama epidemiológico, dado que la más alta mortalidad se registran en los países más pobres. El mapa epidemiológico donde se evidencia su distribución según la geografía, se superpone al mapa de la pobreza, apareciendo Zambia, Tanzania, Malawi, Zimbawe en África Oriental como los países donde se da una mayor incidencia.

En América del Sur y el Caribe, los países con la mortalidad más alta por ésta neoplasia ejemplificadora, es decir el cáncer de cérvix, son Haití, Bolivia, Paraguay, Perú, Nicaragua y El Salvador, que, no por casualidad, tienen el Producto Bruto Interno per cápita más bajo de la zona ${ }^{(4,8,27)}$. La figura también se repite en nuestro país; y en el caso de Lima Metropolitana, su "Registro poblacional de neoplasias, período 1990-1997" encontró las más altas tasas poblacionales de cáncer de cérvix, reportando cifras que van de 29,9 a 39,6 por 100000 habitantes. En éste reporte los distritos de Comas, El Agustino, Carabayllo, Villa El Salvador, Villa María del Triunfo, San Juan De Lurigancho, en los que entre 48 y $59 \%$ de hogares tienen un nivel socioeconómico pobre y muy pobre, fueron los más afectados con ésta patología ${ }^{(15)}$. 
Albújar, et al. hallaron en la ciudad de Trujillo, que la neoplasia de cérvix era la más frecuente, presentando 43,2 casos por cada 100000 habitantes, reportado en el "Registro de Cáncer de Trujillo 19962002" (4,22); y demostrándose por tanto en éstos registros poblacionales que el cáncer de cérvix está asociado a la pobreza ${ }^{(5,12,17)}$. En nuestro Perú, el quinto lugar en cuanto a carga de enfermedad, lo constituyen las neoplasias, siendo mayor la de estómago, las leucemias y la de cérvix. Para el año 2008, los años de vida saludable perdidos (AVISA) por la patología en estudio, fue de 418960 , constituyendo el $8 \%$ de la carga de enfermedad ${ }^{(1,24)}$.

Según Wilkinson y Marmot en su libro "Social Determinants of Health. The Solid Facts" (20), debemos garantizar que menos personas estén en desventaja social y de darse, estas deben ser menos graves. Las políticas públicas delosestadosygobiernos, enelcampo de la empleabilidad, el acceso a vivienda y a educación tienen un efecto directo sobre el estado de salud de sus pobladores ${ }^{(20)}$. Quedando entoncesestablecido que el Gobierno y el Estado tienen la obligación de plantear políticas que establezcan nexos intersectoriales para enfrentar los problemas de salud. Es decir la solución nunca podrá darse solamente desde el sector, toda vez que se tiene que atacar la causa primaria o la causa de la causa de la enfermedad.

Analizando las variables en estudio y como bien dice Wilkinson y Marmot en su libro "Social Determinants of Health. The Solid Facts" (20) compartimos sus ideas sobre los determinantes Sociales, cuando dice que los procesos de exclusión social y el grado de privación relativa en una sociedad tienen consecuencias significativas en salud. El daño se extiende a los procesos mentales y psicológicos de vivir en la pobreza ${ }^{(20)}$. Como salubristas, compartimos las afirmaciones de AgisTsouros (20), quien hace el llamado a los decisores y a los profesionales de la salud pública, para enfrentar los determinantes sociales de la salud y llevarlos más allá de los límites meramente académicos; apoyando dichas decisiones en investigaciones e intervenciones demostradas, es decir en evidencia clara.

Hoy asistimos a un crecimiento enorme de la información, con el desarrollo de investigaciones especializadas y la aparición de tendencias académicas que marcan el rumbo de la gestión en salud, lo que obliga y hace imprescindible realizar las decisiones con un enfoque netamente técnico. Ello posibilitará un uso eficaz y eficiente de los escasos recursos económicos de los países pobres y en vías de desarrollo en el campo sanitario, considerando además que el cáncer es una enfermedad de alto costo. Si está enfermedad ataca a cualquier persona de clase media, la volverá automáticamente pobre, en cualquier parte del mundo, entonces su padecimiento de parte de las poblaciones pobres es, además de penosa, desastrosa y angustiante; aniquilando no solamente al paciente, sino a la familia completa de la que forma parte.

Es necesario por lo tanto que los gobiernos tomen conciencia de su rol en el enfrentamiento de esta enfermedad de alto costo, haciéndose muy necesario la implementación de programas sanitarios contra el cáncer, disponiendo además presupuestos, que podrían ser parte de las políticas de aseguramiento universal, particularmente en Latinoamérica y el Caribe.

Finalmente, dice algo que asumimos como una conclusión máxima, ya que junta nuestra experticia en epidemiología clínica y gestión en salud pública: "Nadie espera que la ciencia sea negra o blanca, sino que sea accesible y que brinde oportunidades para el debate y la toma de decisiones con conocimiento de causa" (20) y en ese sentido desarrollamos la presente investigación. Se concluye, por tanto, que existe una fuerte correlación directa, estadísticamente significativa entre la vulnerabilidad por cáncer y la pobreza en el Perú en el año 2013.

\section{REFERENCIAS BIBLIOGRÁFICAS}

1. César W, Rolando D. Análisis de la Situación del Cáncer en el Perú, 2013. Lima: Ministerio de Salud. Dirección General de Epidemiología; 2013.

2. Instituto Nacional de Estadística e Informática; 2013. Evolución de la pobreza monetaria en el Perú al 2013. Lima: International Agency for Research on Cancer. GLOBOCAN 2008. Lyon: HO/IARC; 2010 [Internet] [Consultado 2015 Sep 01] Disponible en: http://www.iarc. $\mathrm{fr} / \mathrm{en} / \mathrm{media}$-centre/iarcnews/2010/globocan2008.php

3. Solidoro A. Pobreza, Inequidad y Cáncer. Acta Médica Peruana. 2010; 27(03): 204-206.

4. Gutierrez C, Alarcón E. Nivel de pobreza asociado al estadio de gravedad del cáncer ginecológico. An Fac medicina. Lima, 2008; 69(4): 239 - 43.

5. Benites $V$, Rodríguez $\mathrm{Y}$, Mezones-Holguín $\mathrm{E}$. Determinantes sociales del cáncer de cuello uterino. Lima: INS-UNAGESP, Instituto Nacional de Salud; 2013.

6. Solidoro A. El Cáncer Gástrico y la pobreza [Internet] [Consultado 2015 Octubre 9] Disponible en: http://www. iol.com.pe/index.php?option=com_content\&view=article\&id=34:el-cancer-gastrico-y-la-pobreza\&catid=6: articulos\&ltemid $=6$

7. Samuel A. Inequidad y Cáncer: una revisión conceptual. Revista Facultad Nacional de Salud Pública. 2009; 27(3): 341-348. 
8. Miller H. Cáncer y Pobreza [Internet]. [Visualizado 2015 Octubre 10]. Disponible en: http://prodavinci. com/2011/08/06/actualidad/cancer-y-pobreza-porhenry-i-miller/

9. Chan M, Armano Y. En África, el cáncer cobra más vidas por la pobreza [Internet] [Consultado 2015 Ago 30] Disponible en: http://www.who.int/mediacentre/news/ releases/2011/cancer-report- 20115273/es/

10. Organización Mundial de la Salud. La batalla mundial contra el cáncer no se ganará únicamente con tratamiento [Internet] [Consultado 2015 Nov 01] Disponible en: http://www.who.int/mediacentre/news/releases/2014/ cancer-report-20140203/es/

11. Purilla S., Ubillús M. Correlación entre el Cáncer Ginecológico y el Nivel socioeconómico. Instituto Nacional de Enfermedades Neoplásicas 2010-2012. Universidad Nacional Federico Villarreal. Facultad de Medicina Hipólito Unanue. Tesis para optar el Grado de Médico Cirujano. Lima; 2015.

12. Declaran de Interés Nacional La Atención Integral del Cáncer y Mejoramiento del Acceso a los Servicios Oncológicos en el Perú y dictan Otras Medidas. Publicado en el diario oficial El Peruano, Decreto Supremo N ${ }^{\circ} 009$ 2012-SA, (3 de noviembre del 2012).

13. Paul Goss, et al. The Lancet Oncology Commission Planning cancer control in Latin America and the Caribbean.The Lancet Oncology, 2013;14(5):391-436.

14. Payet E, Perez P, Poquioma E, Ubillús M. Registro de Cáncer de Lima Metropolitana, Volúmen IV. Estudio de Incidencia y Mortalidad 2004-2005. Lima: Instituto Nacional de Enfermedades Neoplásicas (INEN). Departamento de Epidemiología y Estadística del Cáncer; 2014.

15. Ubillús $M$, et al. Profile Cancer Epidemiology at the Instituto Nacional de Enfermedades Neoplásicas - Perú. 2000 - 2011. 5. International Cancer Control Congress; November; 2013.

16. Instituto Nacional de Enfermedades Neoplásicas. Programa Presupuestal de Prevención y Control del cáncer. DICON - INEN. Lima 2012.

17. National Cancer Institute USA. Definición de cáncer [Internet] [Consultado 2015 Nov 02] Disponible en: http://www.cancer.gov/espanol/cancer/que-es

18. Milward U, Salazar M, Vidaurre T. El Modelo lógico-causal del Cáncer y la Medicina Basada en Evidencia para las Intervenciones Sanitarias del Programa presupuestal de Prevención y Control del Cáncer en el Perú 2013,. VII Congreso Científico Internacional del Instituto Nacional de Salud; Lima: Desarrollado por el Instituto Nacional de Enfermedades Neoplásicas (INEN); 2013.

19. Wilkinson R, Marmot M. Social Determinants of Health. The Solid Facts. ISBN 92-890-1287-0 (OMS, 2003).
20. Rafael M, et al. Global and regional mortality from 235 causes of death for 20 age groups in 1990 and 2010: a systematic analysis for the Global Burden of Disease Study 2010. The Lancet. 2016; 380(9859): 2095-2128.

21. Vidaurre T, et al. Plan Esperanza: Un Modelo para la Prevención y el Control del Cáncer en Perú. Cáncer Control. Lima: INEN; 2015.

22. Salazar MR, Regalado-Rafael R, Navarro JM, Montañez DM, Abugattas JE, Vidaurre T. El Instituto Nacional de Enfermedades Neoplásicas En El Control del Cáncer en el Perú. RevMed Perú Exp Salud Publica. 2013; 30(1): 105-12.

23. Velásquez A. La carga de enfermedad y lesiones en el Perú y las prioridades del plan esencial de aseguramiento universal. Rev. Perú. med. exp. salud pública; 26(2): 222-31.

24. Bastian $E$, et al. Cáncer de mama y nivel socioeconómico el diagnóstico tardío no es el único factor para que mujeres con un estatus más bajo obtengan peores resultados [Internet] [Consultado 2015 Oct 09] Disponible en: http://www.eurekalert.org/pub_releases_ml/2010-03/ aaft-q_2032210.php

25. Organización Panamericana de la Salud. Salud en Sudamérica. Panorama de la situación de salud y de las políticas y sistemas de salud. Washington DC: Organización Panamericana de la Salud; 2012.

26. Tomatis L. Poverty and cancer. IARC Sci Publ. 1997; (138): 25-39.

27. Listado de Enfermedad de Alto Coste de Atención. Financiamiento Seguro de Salud SIS. Fondo Intangible Solidario de Salud, FISSAL. Resolución Ministerial N 3252012/ Ministerio de Salud (MINSA). República del Perú.

28. Instituto Nacional de Estadística e Informática: Encuesta Demográfica y de Salud Familiar. Lima: INEl; 2012.

29. Pagano M, Gauvreau K. Fundamentos de Bioestadística. Segunda Edición. México DF: Kimberlee Thomson Learning;2001.

30. Tutorial de estadística [Internet] [Consultado 2015 Nov 01] Disponible en: https://explorable.com/es/tutorialde- estadistica

31. Hernandez R, Fernandez C, Baptista P. Metodología de la Investigación. México DF: Mcgraw-Hill Interamericana; 2001.

32. Dever A, Epidemiología y Administración de Servicios de Salud. Maryland: Organización Panamericana de la Salud. Organización Mundial de la Salud; 1991.

33. Tamayo J. Estrategias para diseñar y desarrollar proyectos de investigación en Ciencias de la Salud. Lima: Facultad de Medicina de la Universidad Nacional Mayor de San Marcos; 2002.

34. P. Henquin R. Epidemiología y Estadística Para Principiantes. Ciudad Autónoma de Buenos Aires: Corpus Libros Médicos y Científicos; 2013. 